\title{
Tapping the unexplored potential of marine fungi and edible mushrooms for In silico screening of anti-viral bioactive compounds against SARS- $\mathrm{CoV}-2$ for rapid development of nutraceuticals
}

\author{
Amit Kumar Srivastav ${ }^{\mathrm{a}}$, Jyoti Jaiswal ${ }^{\mathrm{a}}$ and Umesh Kumar ${ }^{\mathrm{a}}{ }^{*}$

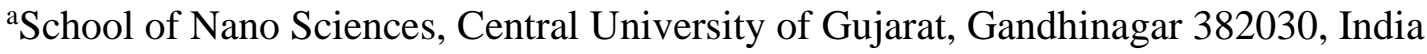

\begin{abstract}
Severe Acute Respiratory Syndrome Coronavirus 2 (SARS- CoV-2) affects human respiratory function that causes COVID-19 disease. COVID-19 has spread rapidly all over the world and became a pandemic within no time. Therefore, it is the need of hour to screen potential lead candidates from natural resources like edible mushrooms and marine fungi. These natural resources are very less explored till now and known to be the source for many medicinal compounds with several health benefits. These medicinal compounds can be easily exploited for the faster development of nutraceuticals for controlling SARS-CoV-2 infections. Our insilico research suggests that bioactive compounds originating from mushroom and marine fungi shows strong potential to interact with ACE2 receptor or main protease of SARS-CoV2 , showing the inhibition activity towards the enzymatic protease. We performed a series of in silico studies for the validation of our results, which includes Molecular docking, drug likeness property investigation by Swiss ADME tools, MD simulation, and thermodynamically stable free binding energy calculation. Overall, these results suggest that Ganodermadiol and Heliantriol $\mathrm{F}$ bioactive compounds originating from edible mushroom has strong potential to be developed as low-cost nutraceutical against SARS-CoV-2 viral infection. The drug candidate isolated from marine fungi and edible mushroom are highly unexplored for the development of potential alternative drug against SARS-CoV-2 virus with minimum side effects. That is why we decided to screen some active metabolites from the marine fungi and mushrooms, which offer some encouraging results. Though our in silico studies of these compounds are showing a promising results against SARS-CoV-2 main protease and ACE2 receptor binding domain, the effectiveness of these bioactive compounds should be further validated by proper clinical trials.
\end{abstract}

Keywords: Coronavirus, Nutraceutical, Bioactive, Molecular docking, MD simulation, Ramachandran plot, Drug likeness, Mushroom, Marine fungi.

*Corresponding authors: Dr Umesh Kumar (umesh.kumar@cug.ac.in) 


\section{INTRODUCTION}

Worldwide recent outbreak of Coronavirus disease 2019 (COVID-19) has caused ongoing public health emergency. This COVID-19 disease is caused by Severe Acute Respiratory Syndrome Coronavirus 2 (SARS- CoV-2). The outburst of SARS-CoV-2 virus is very fast and yet we know very little about this emerging virus. Since the outbreak of this pandemic SARSCoV-2 virus has spread all over the globe within no time. According to the latest update of the $\mathrm{WHO}^{1}$, there are more than 34.8 million cases of COVID-19 worldwide. Over 1 million death have now been reported globally. This novel corona virus was discovered in late December 2019, which caused an epidemic of acute respiratory syndrome in human in Wuhan, China. ${ }^{2,3}$ The Coronaviridae Study Group (CSG), an International Committee on Taxonomy of Viruses, named Severe acute respiratory syndrome related coronavirus 2 and designated as SARS$\mathrm{CoV}-2{ }^{4}$ This novel virus belongs to the $\beta$-coronavirus family a large class of viruses that are widespread in nature. ${ }^{5}$ Coronaviruses are RNA enveloped viruses, having diameter of 80-120 $\mathrm{nm}$ which occurred as universal contagion disease leading to 3 to $4 \%$ mortality rate. ${ }^{5}$ The outbreak of severe acute respiratory syndrome (SARS) CoVs have found to be as zoonotic viruses which can be transmitted between human and animals and bats are considered as natural host of SARS-CoV-2 due to genetic similarities. ${ }^{6}$ It includes four structural proteins : Spike(S) , Envelope (E), Membrane (M) and Nucleocapsid (N) which help in recognising the receptor on target cell, thus leading to transmission of infections. ${ }^{7}$ As the antigen is novel for human host, public health faces serious challenges. Unfortunately, no significant progress has been made in managing the disease so far, and patients are treated based on observable and diagnosable symptoms. Several attempts have been made to diagnose, treat and develop vaccine for this new coronavirus. ${ }^{8}$ To deal with this deadly COVID-19 many phytochemical or herbal compounds have been tried and reported ${ }^{9}$ but no promising outcome is achieved. So, this prompted us to study the inhibition of COVID-19 protease by marine fungi and edible mushroom which is widely used for their high nutritional value and may offer fruitful insights to treat this coronavirus.

Therefore, in the present work we have chosen a multitude of antiviral compounds from Mushrooms $^{10-12}$ such as $\beta$ glucan, Velutin, Heliantriol F, Adenosin, Iso-sinensetin, Dimethylgluanosine, Lentinan and also from marine fungi ${ }^{13-15}$ such as Hispolon, Balticolid, Fucan, Galactan, Equisetin, Stachyflin and many more compounds. We have taken the main protease (Mpro) and ACE2 receptor binding domain as active target of this novel corona virus 
protein and screened with different antiviral compounds found in edible mushrooms and marine fungi using molecular docking tools and molecular dynamic simulation.

Promising results were obtained by screening these compounds based on molecular docking interaction and molecular dynamic simulations. Further these results were also investigated for drug likeness property by using SwissADME web tool. ${ }^{16}$ We also calculated free binding energy through thermodynamically stable receptor-ligand interaction mechanism. Therefore, we believe that our study will lay out the platform for rapid development of alternative drugs for controlling this coronavirus with lesser side effects.

\section{Materials and methods}

\subsection{Molecular docking investigation}

Molecular docking studies are significantly used for analysing and predicting the molecular interaction of ligand-receptor complexes. We used Auto Dock v4.2 with the Lamarckian genetic algorithm for molecular docking studies. ${ }^{17,}{ }^{18}$ In the present work, our primary objective for molecular docking studies is to scrutinize the possibilities of binding between the different bioactive compounds of Mushroom and Fungi with respect to the SARS-CoV-2 (Mpro form \& ACE2 receptor structure). The crystal structures of SARS-CoV-2 (PDB code: 5RH4, 6LZG ${ }^{19}$ ) were obtained from the Protein Data Bank (www.pdb.org). The bioactive compounds of mushroom and fungi were taken from the PubChem database. The SDF format were converted to PDB format using PyMOL software. For getting the accurate results, all parameters were kept same for each docking studies. The grid box conformation for each SARS-CoV-2 structure is given in ESI Table 1. The docked conformation with highest binding affinity was analysed. and selected for further analysis. PyMol, Chimera, Discovery studio 2020 software's were used for the analysis.

\subsection{Pharmacokinetics, drug likeness and bioavailability prediction}

The prediction of pharmacokinetics especially ADME, bioavailability and drug likeness property of bioactive compounds from mushroom and fungi were found using SwissADME web tool. ${ }^{16}$ The pioneer Lipinski (Pfizer) rule-of-five was used for the prediction of drug likeness property. Swiss ADME tool predicts bioavailability based on six physicochemical properties such as lipophilicity, molecular weight, polarity, insolubility, flexibility, and instauration to detect drug likeness. The ADME properties like HIA (human gastrointestinal absorption) and BBB (blood-brain barrier) permeation was also predicted and analyzed using BOILED-Egg model. 


\subsection{Simulation}

Molecular Dynamics is a dynamical simulation study, through which the equations of motion for a receptor-ligand complex system are numerically integrated over time. These data are helpful to obtain particle trajectories in phase space. In our study, molecular dynamics simulations were performed using the GROMACS $2020 .{ }^{20}$ The initial input structures were built from the SARS-CoV-2 crystallographic structure and the initial orientation of the bioactive compounds towards the receptor was obtained from the moleculear docking studies. The complex structure of receptor-ligand was immersed in a dodecahedron-shaped box $(\mathrm{x}, \mathrm{y}$, and $\mathrm{z}$ ) with the minimum distance of $1 \mathrm{~nm}$ between the complex structure surface and the box walls, In order to eliminate the contributions of surfaces that affect the physical properties of the system, periodic boundary conditions are imposed. MD simulation was performed with CHARMM36 force field. ${ }^{21}$ The protein complex with selected bioactive compounds was solvated with TIP3P 22 water molecules in a dodecahedral unit cell box. The systems were neutralized by adding counter ions and periodic boundary conditions were applied. The particle mesh Ewald method was used to calculate the long-range electrostatic interactions while the SHAKE algorithm was used to constrain the hydrogen bonds. ${ }^{23}$ NPT and NVT ensemble was used with periodic boundary conditions. ${ }^{24}$ Pressure was fixed at $1 \mathrm{~atm}$, while the temperature was set at $300 \mathrm{~K}$. The particle-mesh Ewald method ${ }^{25}$ was used to evaluate the Coulomb interactions. 2 fs of time step was used in all MD simulations. Initially, water was equilibrated for $200 \mathrm{ps}$ at $300 \mathrm{~K}$ after fixing the SARS-CoV-2 structure and energy minimization of 1000 steps. 1000 steps of energy minimization of the whole system were performed, and further equilibration for $400 \mathrm{ps}$ at $310 \mathrm{~K}$ after releasing the SARS-CoV-2 structure was done. Simulation run was performed upto $100 \mathrm{~ns}$. The trajectory data were saved at every 1 ps to analyse the change in the dynamics of ACE2-RBD and Mpro structural binding interface using the VMD. The root mean square deviation, Ramachandran plot and secondary structure snapshots at every $5 \mathrm{~ns}$ were calculated for simulated systems. We also analysed the free biding energy for the SARS-CoV-2 main protease and ACE2 receptor binding domain docked with selected bio active compounds. These analyses were conducted using MM/PB(GB)SA method. The simulation was performed till $100 \mathrm{~ns}$ for equilibration and stability during MD simulation. For each complex system, 10000 snapshots were extracted at interval of 10 ps along the trajectory. 


\section{Results and Discussion}

\subsection{Molecular Docking}

In recent times, molecular structure based virtual screening widely used for the discovery and screening of novel lead compounds selected targets. In this work, we conducted structural based virtual screening of 43 different known bioactive compounds from Mushroom and Fungi using the autodock vina tools. The docked complexes of all the synthesized compounds were analysed on the basis of highest binding energy values. Molecular docking results of binding affinities with different bioactive compounds of mushroom and fungi with SARS-CoV-2 structure are shown in Table 1.It was observed that all the selected bioactive compounds exhibited significant binding affinities towards SARS-CoV-2 virus (Table 1).Therefore, we selected bioactive compounds with docking scores better than a threshold of $-10 \mathrm{kcal} / \mathrm{mol}$ for further pharmacokinetics, drug likeness, bioavailability and molecular interaction analysis. We selected a total of eight out of 43 bioactive compounds, i.e. Ganodermadiol (-14.6 Kcal/Mol), Clathsterol (-10.5 Kcal/Mol), Isoescin Ia (-11.6 Kcal/Mol), Mirabamides A (-11.3 Kcal/Mol), Neamphamide A (-13.7 Kcal/Mol), Microspinosamide (-16.8 Kcal/Mol), Heliantriol F (-16.9 Kcal/Mol), Lentinan (-11.4 Kcal/Mol) as most probable inhibitors for the ACE2 receptor domain of SARS-CoV-2 virus. Along with the possible inhibitors for the ACE2 receptor, these eight bioactive compounds also shown significant binding affinity towards the Mpro structure of SARS-CoV-2 virus. The docking scores of these eight bioactive compounds were considered more prominent and significant in comparison to the other bioactive compounds of mushroom and fungi. These results indicate the potential of these eight bioactive compounds from mushroom and fungi as inhibitors of SARS-CoV-2 main protease along with the ACE2 receptor. These docking studies suggests that they could be used for the rapid development of anti-viral drugs for managing SARS-CoV-2 infection. Further these compounds were also analysed by SWISS ADME tool for pharmacokinetics, drug likeness and bioavailability prediction. 


\begin{tabular}{|c|c|c|c|}
\hline \multirow{2}{*}{ Sr. No. } & \multirow{2}{*}{ Fungi based Bioactive ingredient } & \multicolumn{2}{|c|}{ Binding Affinity (Kcal/Mol) } \\
\hline & & ACE2 & Mpro \\
\hline 1. & Microspinosamide & -16.8 & -13.7 \\
\hline 2. & Paclitaxel & -14.6 & -13.8 \\
\hline 3. & Neamphamide A & -13.7 & -13.1 \\
\hline 4. & IA (Isoescin Ia) & -11.6 & -11.6 \\
\hline 5. & Mirabamides A & -11.3 & 10.7 \\
\hline 6. & Clathsterol & -10.5 & -10.1 \\
\hline 7. & Carrageenan & -10.2 & -8.4 \\
\hline 8. & Petrosins & -9.7 & -8.1 \\
\hline 9. & HalovirA & -9.3 & -8.5 \\
\hline 10. & Arisugacin A & -9.0 & -9.0 \\
\hline 11. & Crambescidin & -8.9 & -7.4 \\
\hline 12. & Thalassiolins A & -8.9 & -8.9 \\
\hline 13. & Ganomycin I & -8.8 & -6.0 \\
\hline 14. & Velutin & -8.8 & 7.5 \\
\hline 15. & Ganoderic acid $\beta$ & -8.6 & -7.7 \\
\hline 16. & Stachyflin & -8.5 & -7.9 \\
\hline 17. & Laminaran & -8.2 & -7.3 \\
\hline 18. & Hispolon & -7.9 & -6.6 \\
\hline 19. & Sansalvamide & -7.9 & -6.7 \\
\hline 20. & Equisetin & -7.7 & -7.8 \\
\hline 21. & Integric acid & -7.6 & -6.6 \\
\hline 22. & Phomasetin & -7.6 & -7.4 \\
\hline 23. & NRPS-PKS (Tenellin) & -7.4 & -6.8 \\
\hline 24. & $\begin{array}{l}\text { Cyclo(L-Tyr-L-Pro) } \\
\text { (Maculosin) }\end{array}$ & -7.3 & -7.2 \\
\hline 25. & Balticolid & -7.2 & -6.8 \\
\hline 26. & $\omega$-Hydroxyemodin & -7.1 & -6.4 \\
\hline 27. & 4-methylaaptamine & -7.0 & -5.1 \\
\hline 28. & Antrodin A & -7.0 & -6.1 \\
\hline 29. & Polyacetylenetriol & -6.7 & -6.1 \\
\hline 30. & Galactan & -6.3 & -6.3 \\
\hline 31. & Fucan & -5.9 & -6.0 \\
\hline \multicolumn{4}{|c|}{ Mushroom based Bioactive ingredient } \\
\hline 32. & Heliantriol F & -16.9 & -11.9 \\
\hline 33. & Ganodermadiol & -14.6 & -11.1 \\
\hline 34. & Lentinan & -11.4 & -11.4 \\
\hline 35. & Ganoderone A & -9.1 & -8.5 \\
\hline 36. & Ganoderic acid GS-1 & -8.9 & -8.8 \\
\hline 37. & Velutin & -8.8 & -7.6 \\
\hline 38. & Ganodermanontriol & -8.3 & -7.6 \\
\hline 39. & iso-sinensetin, & -7.9 & -7.5 \\
\hline 40. & $\beta$ glucan & -7.6 & -6.7 \\
\hline 41. & dimethylguanosine & -7.6 & -7.0 \\
\hline 42. & Colossolactone V & -7.6 & -7.9 \\
\hline 43. & Adenosin & -7.3 & -6.5 \\
\hline
\end{tabular}

Table 1: Docking scores of different bioactive compounds with active site residues of SARSCoV-2 main protease and ACE2 receptor binding domain 


\subsection{Pharmacokinetics, drug likeness and bioavailability prediction}

The result of the Swiss ADME tools exhibited physicochemical and bioavailability characteristics of the of the selected bio active compounds. These results include the Lipinski rules of five (MW, Log P, HBAs and HBDs). As per the Lipinski's rule of five all the screened bioactive compounds are presented in Table 2. Out of all the screened bioactive compounds, only 2 compounds were in accordance with the Lipinski's rule of five exhibiting not more than one violation. Explicitly, from the SWISS ADME tool analysis we can infer that Ganodermadiol and Heliantriol $\mathrm{F}$ are within the acceptable range of Molecular weight(MW), Rotatable bonds(RB), Number of H-bond Donors (HBD), Number of H-Bond acceptors (HBA), Topological Polar Surface Area (TPSA), octanol/water partition coefficient (iLOGP), Number of heavy atoms (nAH) and Molar refractivity (MR). These results indicate that these two compounds are quite specific and acceptable for drug candidate.

\begin{tabular}{|c|c|c|c|c|c|c|c|c|}
\hline \multirow{2}{*}{$\begin{array}{l}\text { Sr. } \\
\text { No. }\end{array}$} & \multirow{2}{*}{$\begin{array}{l}\text { Bioactive } \\
\text { compound }\end{array}$} & \multirow{2}{*}{$\begin{array}{l}\text { Molecular } \\
\text { formula }\end{array}$} & \multicolumn{4}{|c|}{ Lipinski rule of 5 (Swiss ADME) } & \multirow{2}{*}{$\begin{array}{c}\text { No. of } \\
\text { Violations }\end{array}$} & \multirow{2}{*}{$\begin{array}{c}\text { Drug } \\
\text { likeness }\end{array}$} \\
\hline & & & $\begin{array}{c}\text { Mol.Wt } \\
(<500 \\
\text { g/mol })\end{array}$ & $\begin{array}{c}\log \\
\mathrm{P} \\
(<5)\end{array}$ & $\begin{array}{c}\mathrm{H} \\
\text { bond } \\
\text { donor } \\
(<5)\end{array}$ & $\begin{array}{l}\mathrm{H} \text { bond } \\
\text { acceptor } \\
(<10)\end{array}$ & & \\
\hline 1. & Clathsterol & $\mathrm{C}_{39} \mathrm{H}_{64} \mathrm{Na}_{2} \mathrm{O}_{15} \mathrm{~S}_{2}$ & 883.03 & -0.56 & 1 & 15 & 2 & No \\
\hline 2. & Ganodermadiol & $\mathrm{C}_{30} \mathrm{H}_{48} \mathrm{O}_{2}$ & 440.70 & 5.79 & 2 & 2 & 1 & Yes \\
\hline 3. & Heliantriol F & $\mathrm{C}_{30} \mathrm{H}_{50} \mathrm{O}_{3}$ & 458.72 & 5.28 & 3 & 3 & 1 & Yes \\
\hline 4. & IA (Isoescin Ia) & $\mathrm{C}_{55} \mathrm{H}_{86} \mathrm{O}_{24}$ & 1131.26 & -0.46 & 13 & 24 & 3 & No \\
\hline 5. & Lentinan & $\mathrm{C}_{42} \mathrm{H}_{72} \mathrm{O}_{36}$ & 1153.00 & $\begin{array}{c}- \\
11.70\end{array}$ & 23 & 36 & 3 & No \\
\hline 6. & Microspinosamide & $\mathrm{C}_{75} \mathrm{H}_{109} \mathrm{BrN}_{18} \mathrm{O}_{22} \mathrm{~S}$ & 1726.74 & -1.18 & 17 & 23 & 3 & No \\
\hline 7. & Mirabamides A & $\mathrm{C}_{66} \mathrm{H}_{104} \mathrm{ClN}_{13} \mathrm{O}_{21}$ & 1451.06 & -2.08 & 16 & 22 & 3 & No \\
\hline 8. & Neamphamide A & $\mathrm{C}_{75} \mathrm{H}_{125} \mathrm{~N}_{21} \mathrm{O}_{23}$ & 1688.92 & -5.09 & 22 & 22 & 3 & No \\
\hline
\end{tabular}

Table 2: Pharmacokinetics, drug likeness and bioavailability prediction based on Swiss ADME tool

Hence, we focused on these two bioactive compounds namely Ganodermadiol and Heliantriol F for furthers molecular interaction and simulation studies. Bioactive compounds exhibiting desired pharmacokinetic profile could be exploited for rapid development of promising and effective inhibitor for the SARS-CoV-2 with minimum side effects. 


\subsection{Molecular interaction and MM/GBSA analysis}

Molecular interactions in protein-ligand docked complexes can lead to improved understanding of molecular mechanisms in biological systems. Molecular interaction profiling was studied for the Ganodermadiol and Heliantriol F with SARS-CoV-2 Mpro and ACE2 receptor structure. The Ganodermadiol complex exhibited interaction by moderate single and double hydrogen bonds in the active region with HIS540 (3.2 $\AA$ ), THR434 (2.7 $⿱$ A) and ASN290 (3.0 $\AA, 2.6 \AA$ ) residues, respectively. Additional hydrophobic attractions were also recorded within the Ganodermadiol docked complex at residues PHE 438 (2.6 ̊), ILE 291(3.0 ̊). Also, residues like ASP431(2.8 ̊), ASP 367(2.2 Å) show polar interactions. Meanwhile, positive (LYS 441(2.2)) and negative charge interactions (ASP367(1.8)) with residues were also found in the Ganodermadiol docked complex with SARS-CoV-2 ACE2 binding receptor (Fig. 3a, b). Similarly, for the SARS- CoV-2 main protease structure the Ganodermadiol complex displayed moderately two double hydrogen bonds at GLN189 (2.0 А, $2.6 \AA)$ and HIS163 (2.2,3.0). Single hydrogen bond formation was observed at residues HIS 41 (2.5 $)$ ), GLN192 (3.2 $)$ ). The $\pi-\pi$ interaction was formed at PHE140(2.0 ̊), SER146(3.0 ̊) and MET49(2.7 ̊), which shows high hydrophobic interaction probability between receptor and ligand. The interaction profiles of Heliantriol F with SARS-CoV-2 ACE2 binding receptor reflected three single hydrogen

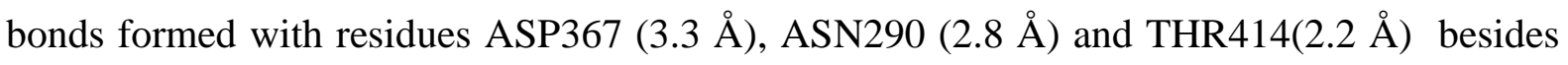
double hydrogen bonds formation at GLU435 (2.1 $\AA$, $2.4 \AA)$. We observed $\pi$ - $\pi$ bond

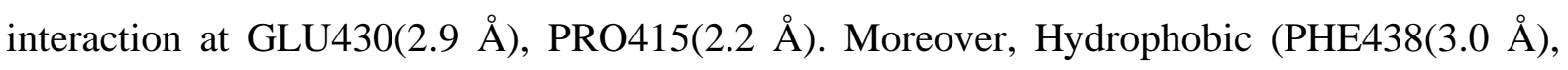

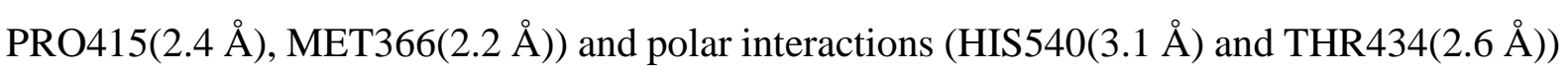
were also logged in the docked complex. LYS541(2.9 $)$ showed positive charge interactions at the active pockets of SARS-CoV-2 ACE2 binding receptor with Heliantriol F. Likewise for SARS- CoV-2 main protease structure, Heliantriol $F$ shows promising $\pi$ - $\pi$ interaction at

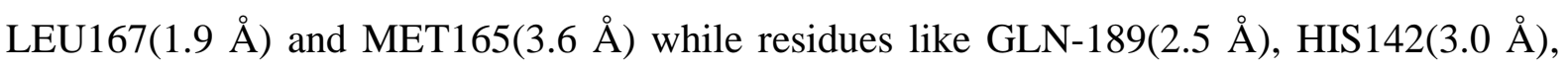
GLU166(1.7 ̊) reflects single hydrogen bonds formation. 


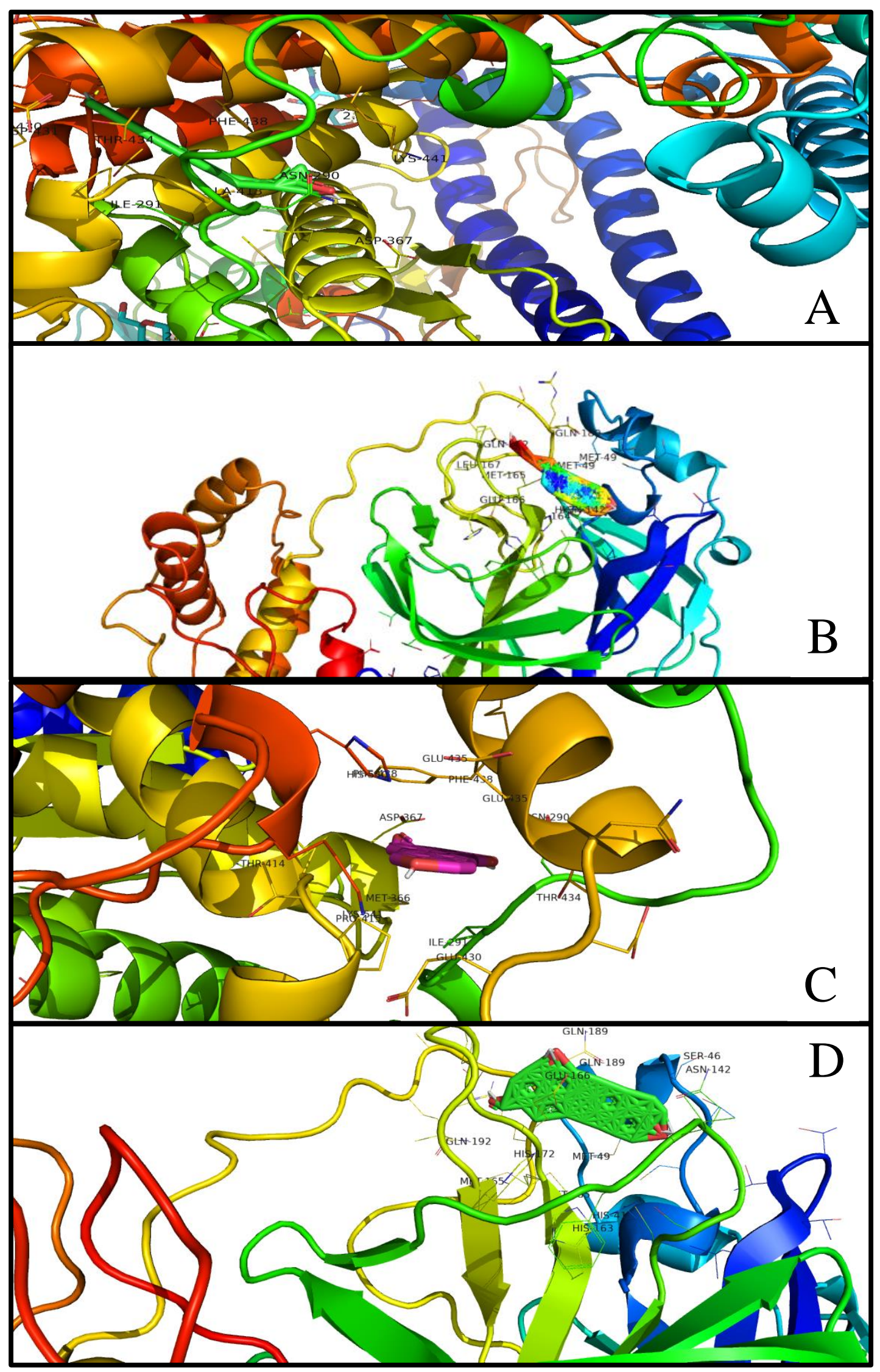

Figure 1: Molecular docked structure of (A) Ganodermadiol with SARS-CoV-2 ACE2 receptor binding domain (B) Ganodermadiol with SARS-CoV-2 Mpro structure (C) Heliantriol F with SARS-CoV-2 ACE2 receptor binding domain (D) Heliantriol F with SARS-CoV-2 Mpro structure. 
Hence, we can infer that the screened Ganodermadiol and Heliantriol F exhibit a strong affinity towards the SARS-CoV-2 main protease and ACE2 receptor. These numerous intermolecular interactions of ligand with receptors predicts that this bioactive compound could be exploited for developing effective protease inhibitors and rapid capturing of coronaviruses. Furthermore, the docked complexes of Ganodermadiol and Heliantriol F with SARS-CoV-2 main protease and ACE2 receptor were also reconfirmed and analysed using MM/GBSA calculations. These results were used to predict the binding affinities of these bioactive ligands with respect to the SARS-CoV-2 main protease and ACE2 receptor. These MM/GBSA free binding energy calculations exhibited negative binding energy values for all four simulated docked complexes, i.e. Ganodermadiol with SARS-CoV-2 ACE2 receptor (-57.89 kcal/ mol), Ganodermadiol with SARS-CoV-2 main protease $(-53.73 \mathrm{kcal} / \mathrm{mol})$. While Heliantriol F showed free binding energy of $-63.28 \mathrm{kcal} / \mathrm{mol}$ and $-46.87 \mathrm{kcal} / \mathrm{mol}$ for SARS-CoV-2 ACE2 receptor and SARSCoV-2 main protease, respectively. (Table 3 )

\begin{tabular}{|c|c|c|}
\hline \multirow{2}{*}{$\begin{array}{c}\text { ENERGIES } \\
\text { (KCAL/MOL) }\end{array}$} & \multicolumn{2}{|c|}{ SARS-COV-2-ACE2 (6LZG) } \\
\hline & Heliantriol F & Ganodermadiol \\
\hline$\Delta \mathbf{E}_{\text {ELECtrostatic }}$ & $-41.72 \pm 0.61$ & $-35.21 \pm 0.58$ \\
\hline$\Delta \mathbf{E}_{\mathrm{VDW}}$ & $-50.67 \pm 0.86$ & $-46.18 \pm 0.94$ \\
\hline$\Delta \mathbf{G}_{\mathbf{G B}}$ & $18.34 \pm 0.16$ & $22.37 \pm 0.82$ \\
\hline$\Delta \mathbf{G}_{\mathrm{SA}}$ & $-9.76 \pm 0.30$ & $-15.25 \pm 0.69$ \\
\hline$\Delta \mathbf{H}$ & $-12.19 \pm 0.10$ & $-12.37 \pm 0.48$ \\
\hline$-\mathbf{T \Delta S}$ & $20.53 \pm 0.09$ & $16.38 \pm 0.27$ \\
\hline$\Delta \mathbf{G}$ & $-63.28 \pm 0.12$ & $-57.89 \pm 0.78$ \\
\hline \multirow{2}{*}{$\begin{array}{c}\text { ENERGIES } \\
\text { (KCAL/MOL) }\end{array}$} & \multicolumn{2}{|c|}{ SARS-CoV-2-Mpro (5RH4) } \\
\hline & Heliantriol F & Ganodermadiol \\
\hline$\Delta \mathbf{E}_{\text {ELECTROSTATIC }}$ & $-36.15 \pm 0.58$ & $-38.24 \pm 0.41$ \\
\hline$\Delta \mathbf{E}_{\text {VDW }}$ & $-41.34 \pm 0.53$ & $-45.74 \pm 0.29$ \\
\hline$\Delta \mathbf{G}_{\mathbf{G B}}$ & $20.81 \pm 0.78$ & $23.19 \pm 0.45$ \\
\hline$\Delta \mathbf{G}_{\mathrm{SA}}$ & $-6.13 \pm 0.19$ & $-9.11 \pm 0.18$ \\
\hline$\Delta \mathbf{H}$ & $-11.34 \pm 0.41$ & $-16.81 \pm 0.26$ \\
\hline$-\mathbf{T} \Delta \mathbf{S}$ & $15.94 \pm 0.06$ & $16.17 \pm 0.79$ \\
\hline$\Delta \mathbf{G}$ & $-46.87 \pm 0.55$ & $-53.73 \pm 0.38$ \\
\hline
\end{tabular}

Table 3: Free binding energy for SARS-CoV-2 spike rbd ACE2 receptor simulated complex and Mpro simulated complex with highest docked score bioactive compounds. 
These free binding energy results confirms the stronger binding affinity of these two bioactive compounds against SARS-CoV-2 main protease and ACE2 receptor, which shows a stronger inhibition of these two bioactive compounds against the SARS-CoV-2 main protease and stronger affinity with ACE2 receptor binding domain. In addition, $\Delta \mathrm{E}_{\text {electrostatic, }} \Delta \mathrm{E}_{\mathrm{vdw}}, \Delta \mathrm{G}_{\text {solv }}$ and solvent-accessible surface area (SASA) were also analysed for the selected Ganodermadiol and Heliantriol F complexed with SARS-CoV-2 viral protein. Our data indicates that, depending on the ligand $\Delta \mathrm{E}_{\text {electrostatic, }} \Delta \mathrm{E}_{\mathrm{vdw}}$ contributed the most to the stability of the Ganodermadiol and Heliantriol F complexes with the SARS-CoV-2 viral protein. (Table 3). Hence, Ganodermadiol and Heliantriol $\mathrm{F}$ was concluded as the most promising bioactive inhibitor for the SARS-CoV-2 virus, and further analysed along with the SARS-CoV-2 main protease and ACE2 receptor binding domain using molecular dynamics simulation.

\subsection{Simulation}

Molecular dynamics (MD) simulation studies predict the conformational and structural molecular interaction based on the receptor-ligand dynamic behaviour. Information like molecular Interaction, pharmacokinetics, drug likeness and bioavailability prediction further validated by molecular dynamics simulation studies. Herein, the stability of the selected receptor-ligand complexes was evaluated using $100 \mathrm{~ns}$ MD simulation in terms of root mean square deviation (RMSD), Ramachandran plot, secondary structure validation and 3D structural analysis. The RMSD analysis of $\mathrm{C} \alpha$ and backbone atoms in SARS-CoV-2 main protease and ACE2 receptor complexed with Ganodermadiol and Heliantriol F ligands shows stable and acceptable deviations during the $100 \mathrm{~ns}$ MD simulation (Fig. 2). Interestingly, the final fluctuation in RMSD values of Heliantriol F was logged $3.65 \AA$ and $2.15 \AA$ for SARSCoV-2 main protease and ACE2 receptor complexes, respectively. Whereas the Ganodermadiol also showed a stable and maximum variation in RMSD of $5.40 \AA$ and $3.88 \AA$ for SARS-CoV-2 ACE2 binding receptor and SARS-CoV-2 main protease complexes, respectively. Analysing RMSD data we can easily observe that Heliantriol F complexed with SARS-CoV-2 structure shows stable behaviour approx. at 20 ns simulation. Similarly, Ganodermadiol complexed system was relaxed with the acceptable deviations after $15 \mathrm{~ns}$ of simulation process and final deviation was recorded at less than $6 \AA$ and $4 \AA$ for ACE2 binding receptor and main protease respectively at $100 \mathrm{~ns}$ simulation. 


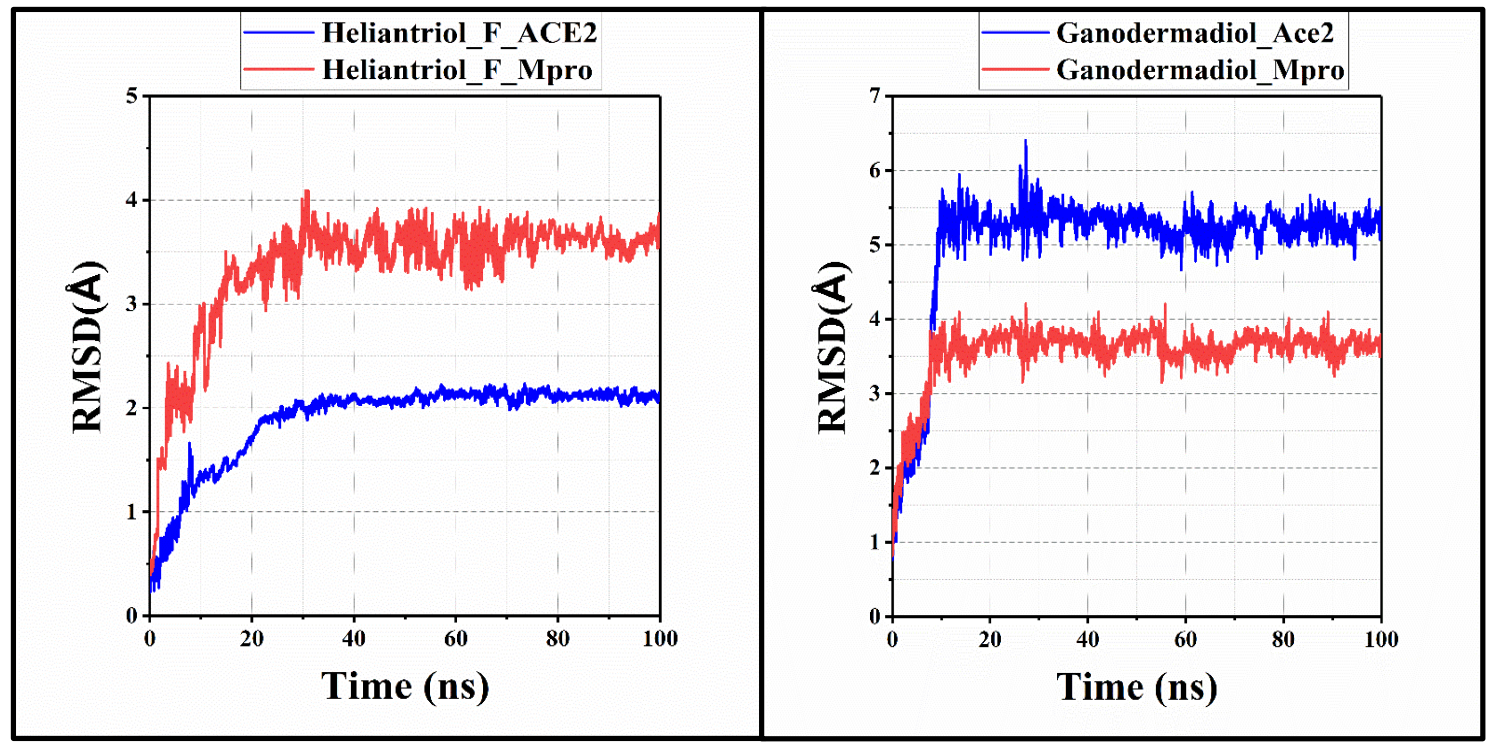

Figure 2: Root mean square deviation graph for Ganodermadiol and Heliantriol F with SARS-

CoV-2 Mpro and ACE2 spike receptor binding domain

These observations suggested the stability of all four simulated complexed structure, which was further validated by the Ramchandran plot. The 2D diagram of all four simulated complexed structure was shown in Fig. 3

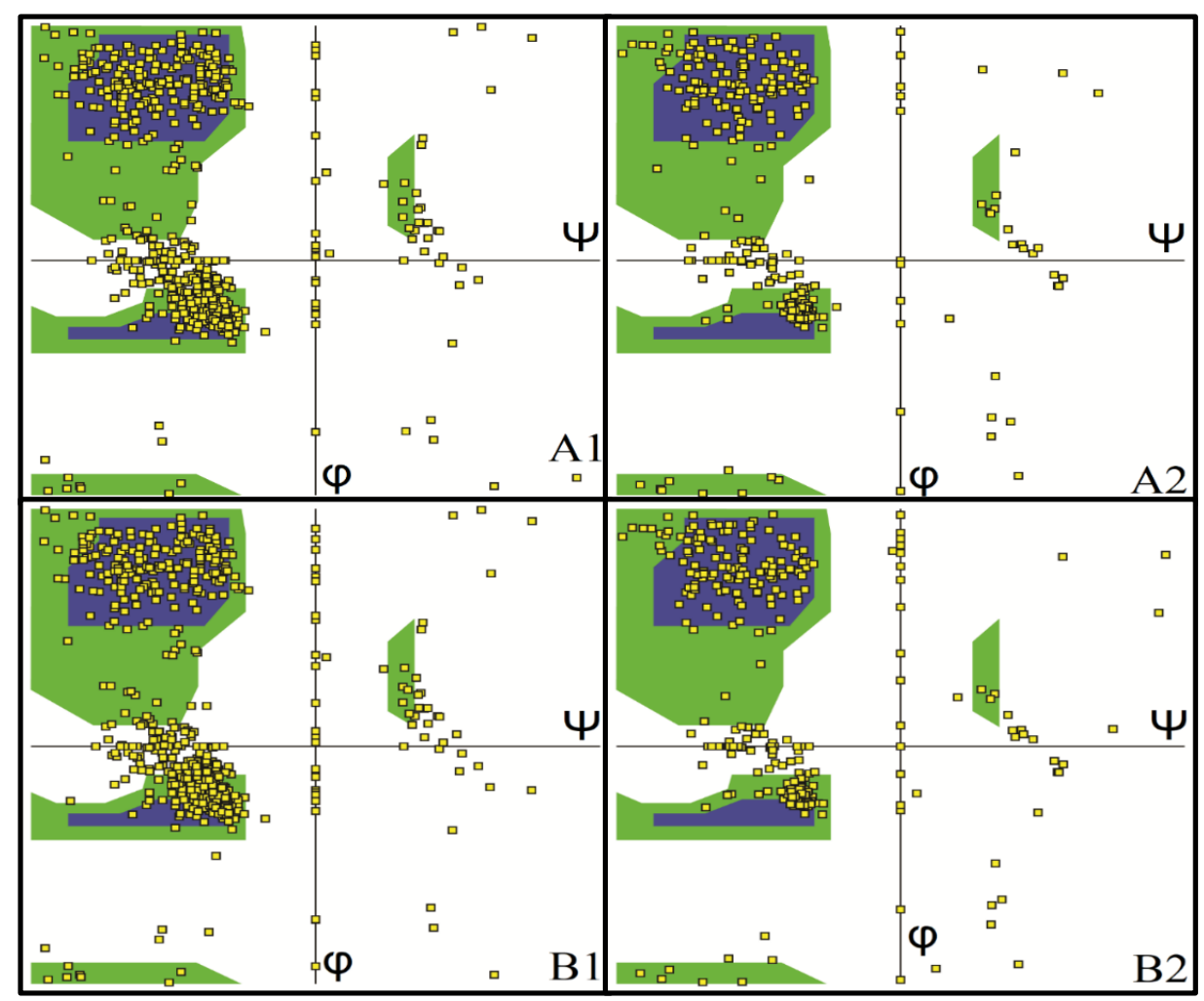

Figure 3: Ramachandran plot (A1) Heliantriol F with SARS-CoV-2 ACE2 receptor binding domain (A2) Heliantriol F with SARS-CoV-2 Mpro structure. (B1) Ganodermadiol with SARS-CoV-2 ACE2 receptor binding domain (B2) Ganodermadiol with SARS-CoV-2 Mpro structure. 
The Ramachandran plot is helpful for analysing the structural or conformational changes in the simulated system. We analyzed and confirmed the changes in the conformational structure of all four SARS-CoV-2-ligand (Ganodermadiol and Heliantriol F) simulated complexes. These conformational changes in terms of backbone dihedral angles $\psi$ against $\varphi$ of the amino acid residues occur for the energetically activated region (Fig 3). Validation of Ramachandran plot for all four simulated complex system were performed with the PROCHECK server. ${ }^{26}$ It revealed that simulated Heliantriol F with ACE2 receptor has 90.5\% residues of SARS-CoV2 receptor

\begin{tabular}{|c|c|c|c|c|c|c|}
\hline \multirow{2}{*}{$\begin{array}{c}\text { Phytochemicals/ } \\
\text { active ingredient }\end{array}$} & $\begin{array}{c}\text { Most } \\
\text { Favoured } \\
\text { Regions[A, } \\
\text { B,L] } \\
\text { (in } \\
\text { percentage) }\end{array}$ & $\begin{array}{c}\text { Additional } \\
\text { Allowed } \\
\text { Regions[a,b,1 } \\
\text {,p] } \\
\text { (in } \\
\text { percentage) }\end{array}$ & $\begin{array}{c}\text { Generousl } \\
\text { y Allowed } \\
\text { Region } \\
\text { (in } \\
\text { percentage } \\
\text { ) }\end{array}$ & $\begin{array}{c}\text { Disallowe } \\
\text { d Regions } \\
\text { (in } \\
\text { percentag } \\
\text { e) }\end{array}$ & $\begin{array}{c}\text { G- } \\
\text { Facto } \\
\mathrm{r}\end{array}$ \\
\hline \multirow{2}{*}{ Ganodermadiant F } & ACE2 & 90.5 & 9.1 & 0.1 & 0.3 & 0.05 \\
\cline { 2 - 8 } & M pro & 86.6 & 10.2 & 1.6 & 1.6 & -0.12 \\
\cline { 2 - 8 } & ACE2 & 90.9 & 8.7 & 0.1 & 0.3 & 0.09 \\
\hline
\end{tabular}

Table 4: Validation report of Ramachandran Pot based on PROCHECK server for most favoured regions $[\mathrm{A}, \mathrm{B}, \mathrm{L}]$, Additional allowed regions $[\mathrm{a}, \mathrm{b}, 1, \mathrm{p}]$, Generously allowed region, Disallowed regions and G-factors.

were in the most favoured regions $[\mathrm{A}, \mathrm{B}, \mathrm{L}]$, followed by $9.1 \%$ in additional allowed regions $[a, b, 1, p], 0.1 \%$ in generously allowed region $[\sim a, \sim b, \sim 1, \sim p]$ and $0.3 \%$ in the disallowed regions. Overall, G factor for the Heliantriol F complexed with ACE2 receptor structure was 0.05 (Table 4). This background data was further cross verified by analysing the secondary structure of all four simulated complex system. The complete result of Ramachandran plot is tabulated in Table 4. The secondary structure of all Ganodermadiol and Heliantriol F simulated complexes was analysed through the STRIDE $^{27}$ (secondary structure identification interface) program to see the conformational changes in the amino acid residues of SARS-CoV-2 ACE2 receptor and Mpro structure as predicted in Ramachandran plot (Figure 4(A), 4(B)). Significantly, we observed the conformational and structural changes in secondary structure during the MD simulation process. These changes are prominently shown in complexed structure of Ganodermadiol and Heliantriol $F$ with respect to the SARS-CoV-2 Mpro form and ACE2 receptor-binding domain. These changes can be seen in terms of alpha-helix, extended configuration, isolated beta bridge, turn, coil, 3-10 helix, Pi-helix. 
Secondary structure of simulated SARS-CoV-2 with ACE2 receptor Chain A

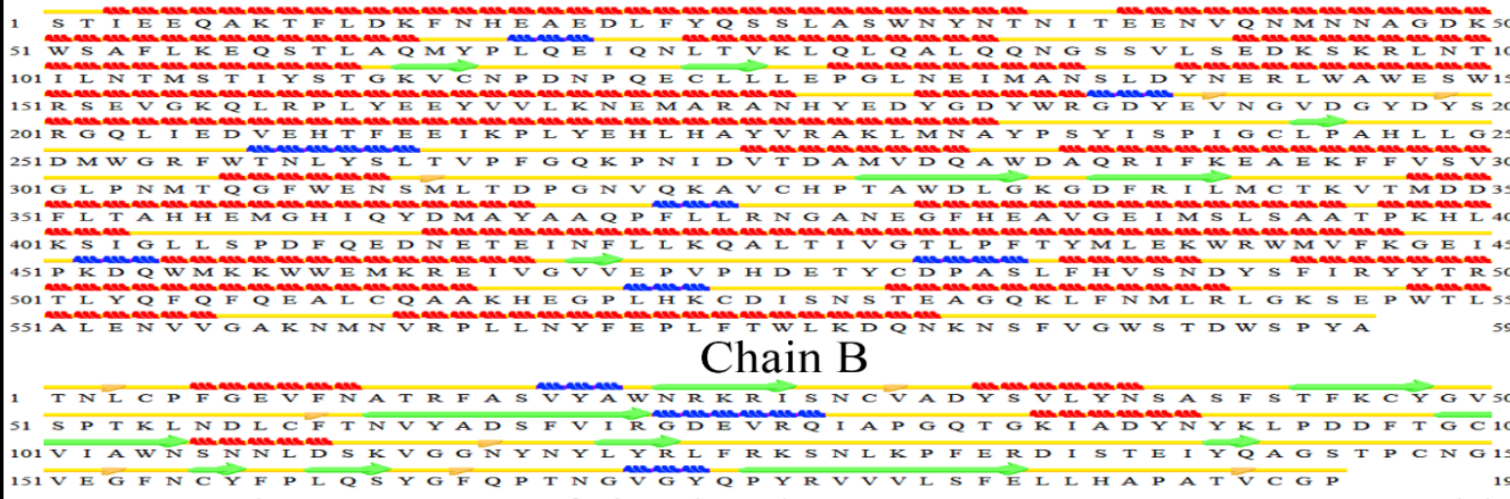

Secondary structure of simulated SARS-CoV-2 ACE2 receptor with Heliantriol F

Chain A

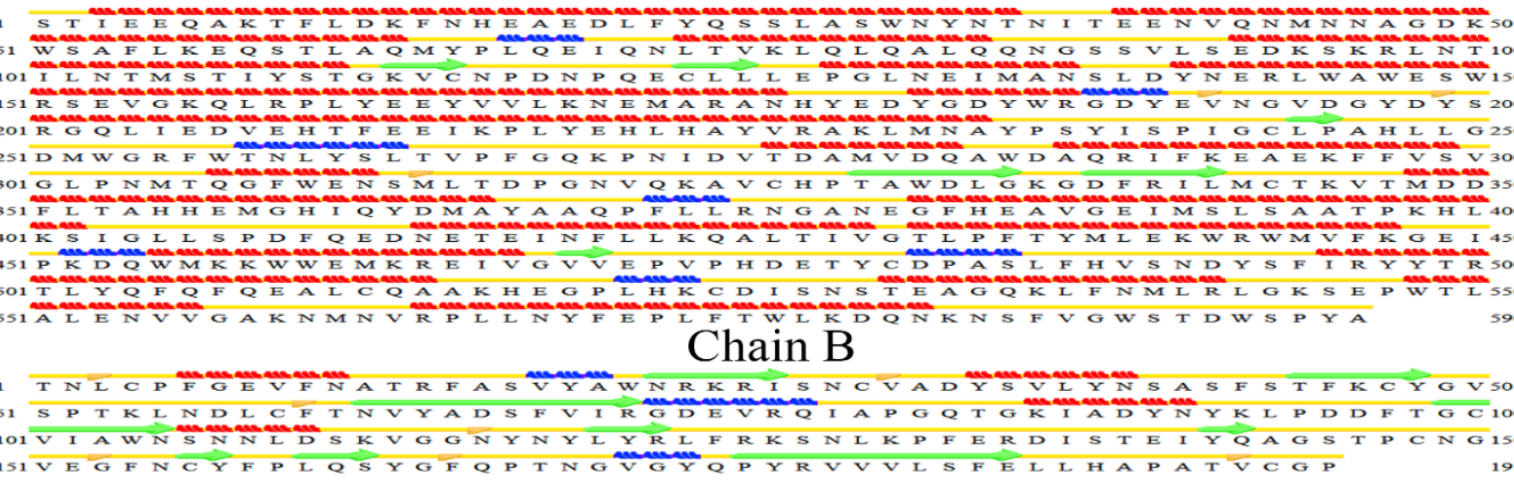

Secondary structure of simulated SARS-CoV-2 ACE2 receptor with Ganodermadiol

Chain A

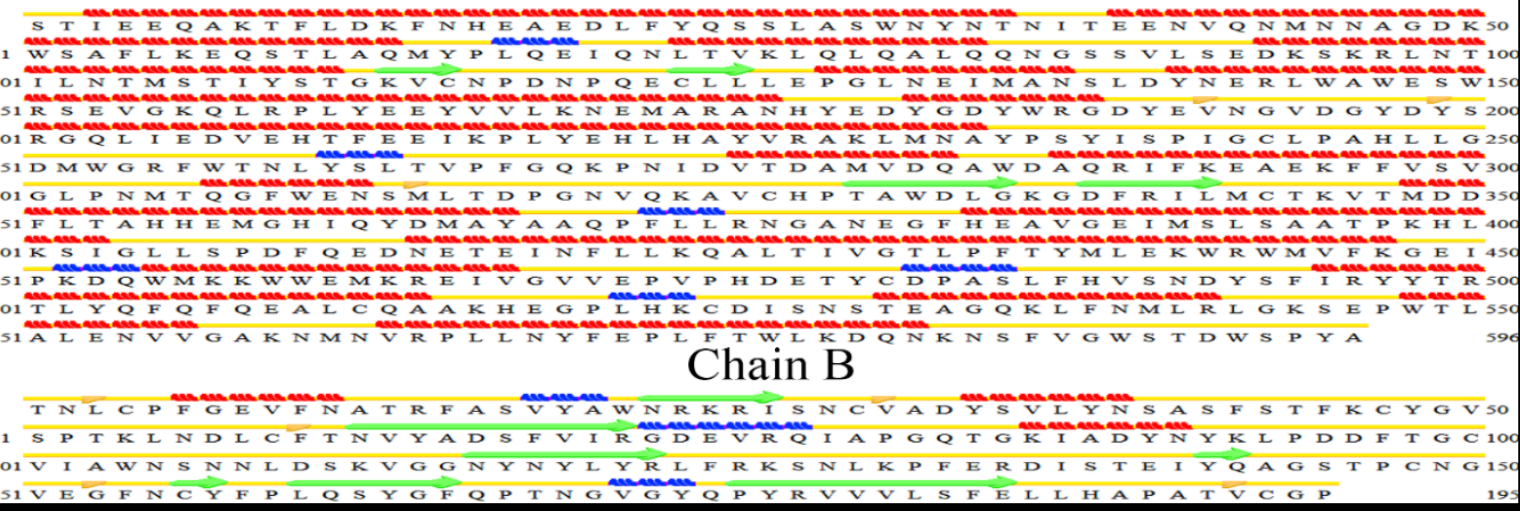

Figure 4(A): Comparison of secondary structure of SARS-CoV-2 spike rbd ACE2 receptor with simulated SARS-CoV-2 ACE2 receptor in complexation with Ganodermadiol and Heliantriol F 


\section{Secondary structure of SARS-CoV-2 Mpro form}

Chain A

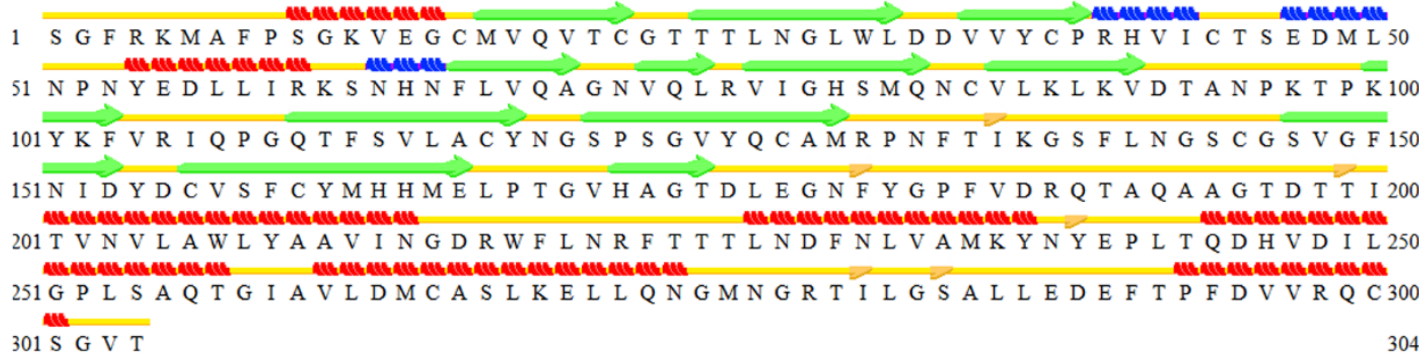

\section{Secondary structure of simulated SARS-CoV-2 Mpro form with Heliantriol F}

Chain A

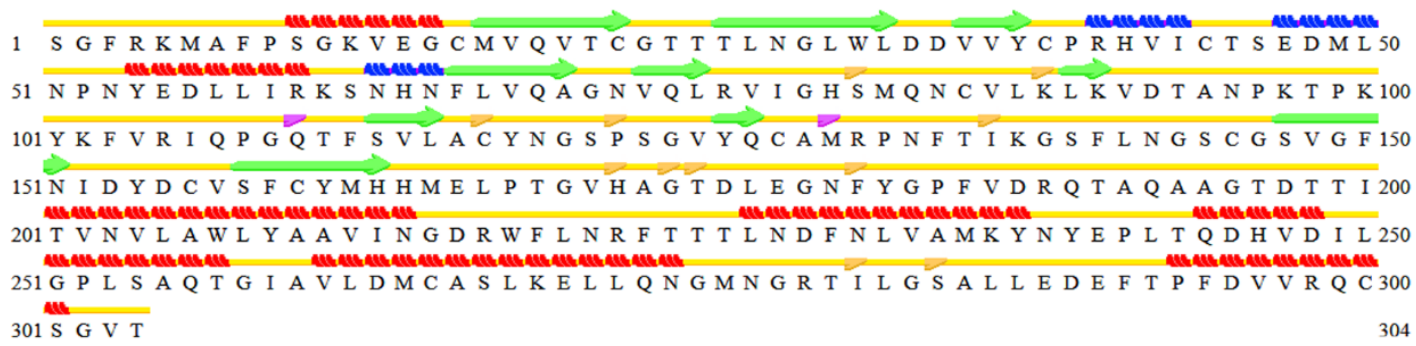

Secondary structure of simulated SARS-CoV-2 Mpro form with Ganodermadiol

Chain A

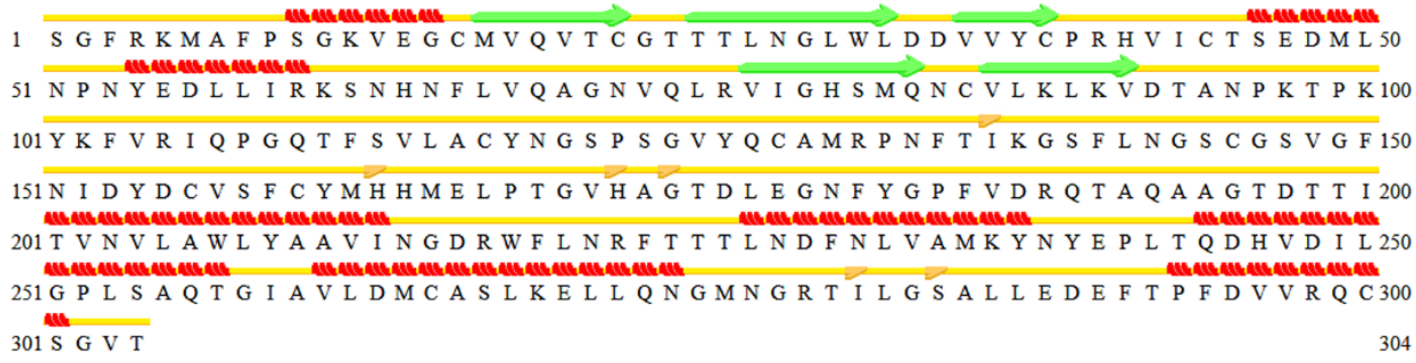

Figure 4(B): Comparison of secondary structure of SARS-CoV-2 Mpro with simulated SARSCoV-2 Mpro in complexation with Ganodermadiol and Heliantriol F

\subsection{Structural analysis of the MD trajectories}

The trajectory files of $100 \mathrm{~ns}$ for all four simulated model were converted to the pdb structure through the GROMACS software and visualized through the VMD program. For analysis and visualization of the coordinates of complexed structure, the MD simulation was carried out with the integration time stamp of 2 ps. Visualization and analysis of simulated structure was 
carried out using VMD and GROMACS software in the CHARMM36 force field. We applied leapfrog integration and Verlet function method to generate the topology and trajectory files. To shed insights into the interaction mechanism of Ganodermadiol and Heliantriol $F$ with respect to the SARS-CoV-2 Mpro and ACE2 receptor-binding domain, we analysed the 3D structure of each complexed simulated structure. The 3D structure of all four simulated receptor-ligand is shown in Figure 5 (A) and 5 (B).

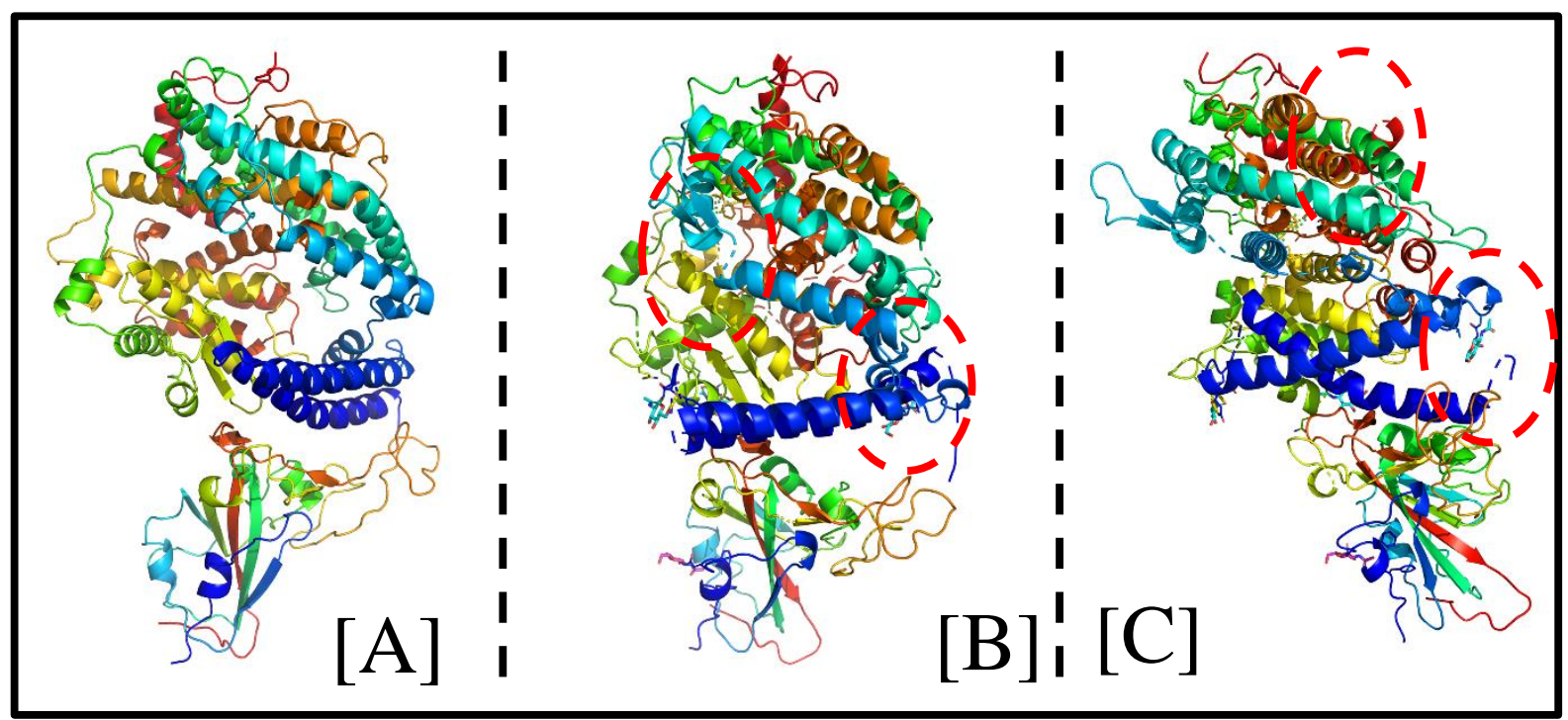

Figure 5(a): 3D structure of SARS-CoV-2 spike rbd ACE2 receptor[A] with simulated SARSCoV-2 ACE2 receptor in complexation with Ganodermadiol [B] and Heliantriol F [C].

In Figure 5, differences can be seen easily in terms of secondary structure like alpha-helix, extended configuration, isolated beta bridge, turn, coil, 3-10 helix, Pi-helix, as well as the hydrogen bonding of protein receptor residues with the Ganodermadiol and Heliantriol F. The conformational changes can also be seen in ACE2 receptor binding domain after conjugation with both ligands. Similarly, the changes in the 3D structure of SARS-CoV-2 main protease can be easily identified in fig.5(b). These Conformational changes confirm the prominent and effective binding interaction between SARS-CoV-2 main protease and ACE2 receptor with Ganodermadiol and Heliantriol F ligand.

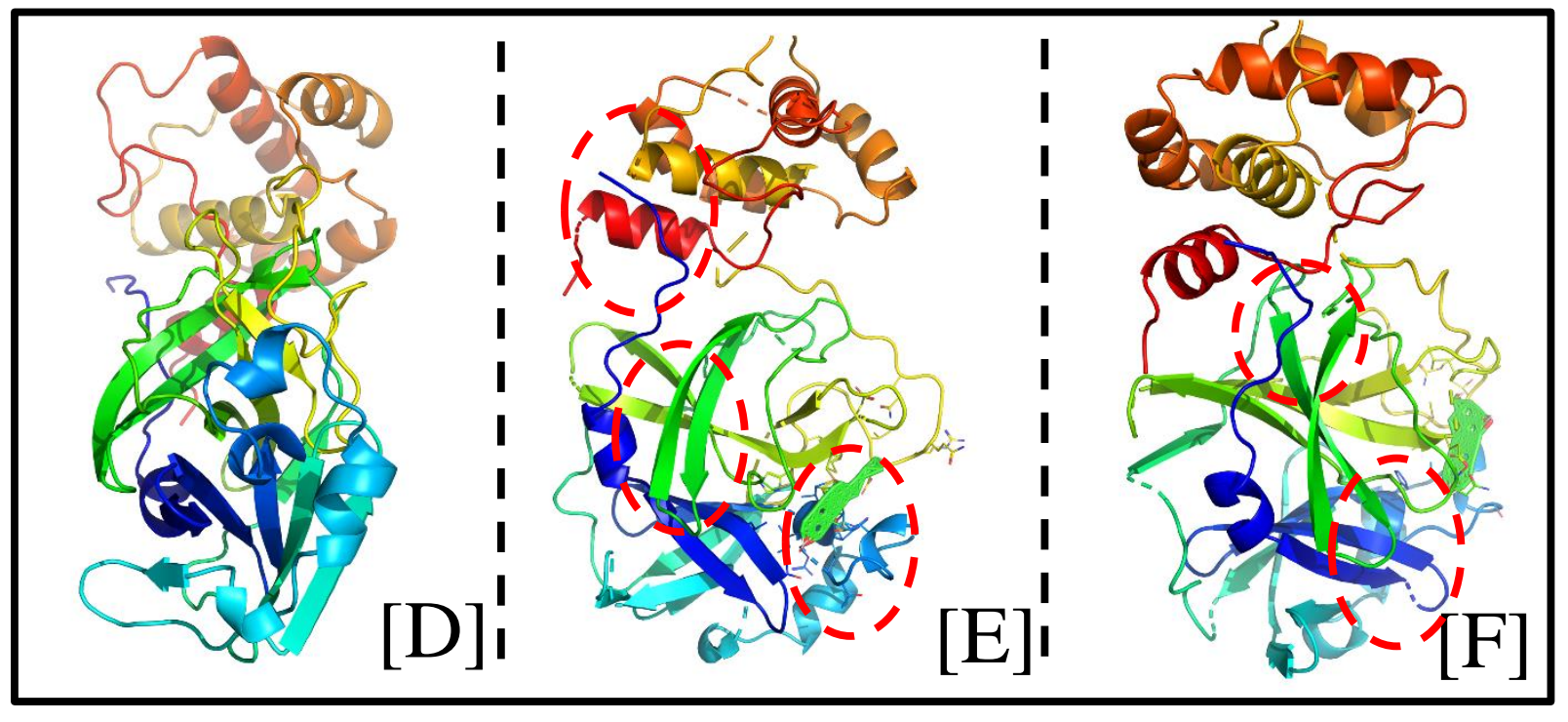


Figure 5(b): Comparison of secondary structure of SARS-CoV-2 Mpro [D] with simulated SARS-CoV-2 Mpro in complexation with Ganodermadiol[E] and Heliantriol F[F].

\section{Conclusion}

Our in-silico assessments suggest that Ganodermadiol and Heliantriol $\mathrm{F}$ have sufficient attributes to be developed as strong inhibitor of the SARS-CoV-2 main protease and rapid capturing of coronaviruses via strong binding with ACE2 receptor binding domain. Our study provides the detail insight of molecular interaction along with the thermodynamic stability of receptor-ligand interaction mechanism. Ganodermadiol and Heliantriol F shows a stable and conformational flexibility with promising efficacy during the molecular interaction in thermodynamic terms with different SARS-CoV-2 motifs. This strong binding behaviour can be used for the rapid development of nutraceutical for controlling or managing SARS-CoV-2 viral infection. Our investigation strongly supports that the natural bioactive compounds like Heliantriol $\mathrm{F}$ and Ganodermadiol from edible mushrooms/marine fungi have promising therapeutic potential which can be further exploited for the rapid development of nutraceutical against the SARS-CoV-2 virus. Although our results are very promising, it is very essential that these data need to be validated by high quality clinically research. This will shed light on the full potential of bioactive compounds from mushroom and marine fungi as source for novel antiviral agents.

\section{Conflicts of interest}

There are no conflicts of interest between authors.

\section{Acknowledgements}

Amit Kumar Srivastav and Jyoti Jaiswal would like to thank University Grant Commission (UGC) for providing non-NET fellowship.

\section{References}

1. World Health, O. Novel Coronavirus (2019-nCoV): situation report, 19; World Health Organization: Geneva, 2020-02-08, 2020.

2. Zhou, P.; Yang, X.-L.; Wang, X.-G.; Hu, B.; Zhang, L.; Zhang, W.; Si, H.-R.; Zhu, Y.; Li, B.; Huang, C.-L.; Chen, H.-D.; Chen, J.; Luo, Y.; Guo, H.; Jiang, R.-D.; Liu, M.Q.; Chen, Y.; Shen, X.-R.; Wang, X.; Zheng, X.-S.; Zhao, K.; Chen, Q.-J.; Deng, F.; Liu, L.-L.; Yan, B.; Zhan, F.-X.; Wang, Y.-Y.; Xiao, G.-F.; Shi, Z.-L., A pneumonia outbreak associated with a new coronavirus of probable bat origin. Nature 2020, 579 (7798), 270-273. 
3. Wu, F.; Zhao, S.; Yu, B.; Chen, Y.-M.; Wang, W.; Song, Z.-G.; Hu, Y.; Tao, Z.W.; Tian, J.-H.; Pei, Y.-Y.; Yuan, M.-L.; Zhang, Y.-L.; Dai, F.-H.; Liu, Y.; Wang, Q.-M.; Zheng, J.-J.; Xu, L.; Holmes, E. C.; Zhang, Y.-Z., A new coronavirus associated with human respiratory disease in China. Nature 2020, 579 (7798), 265-269.

4. Gorbalenya, A. E.; Baker, S. C.; Baric, R. S.; de Groot, R. J.; Drosten, C.; Gulyaeva, A. A.; Haagmans, B. L.; Lauber, C.; Leontovich, A. M.; Neuman, B. W.; Penzar, D.; Perlman, S.; Poon, L. L. M.; Samborskiy, D. V.; Sidorov, I. A.; Sola, I.; Ziebuhr, J.; Coronaviridae Study Group of the International Committee on Taxonomy of, V., The species Severe acute respiratory syndrome-related coronavirus: classifying 2019-nCoV and naming it SARS-CoV-2. Nature Microbiology 2020, 5 (4), 536-544.

5. Zhu, N.; Zhang, D.; Wang, W.; Li, X.; Yang, B.; Song, J.; Zhao, X.; Huang, B.; Shi, W.; Lu, R.; Niu, P.; Zhan, F.; Ma, X.; Wang, D.; Xu, W.; Wu, G.; Gao, G. F.; Tan, W., A Novel Coronavirus from Patients with Pneumonia in China, 2019. The New England journal of medicine 2020, 382 (8), 727-733.

6. Ye, Z.-W.; Yuan, S.; Yuen, K.-S.; Fung, S.-Y.; Chan, C.-P.; Jin, D.-Y., Zoonotic origins of human coronaviruses. International Journal of Biological Sciences 2020, 16 (10), 1686-1697.

7. Wang, L.; Wang, Y.; Ye, D.; Liu, Q., Review of the 2019 novel coronavirus (SARSCoV-2) based on current evidence. Int J Antimicrob Agents 2020, 55 (6), 105948-105948.

8. Li, G.; De Clercq, E., Therapeutic options for the 2019 novel coronavirus (2019-nCoV). Nature reviews. Drug discovery 2020, 19 (3), 149-150.

9. Amit Kumar, S.; Sanjeev Kumar, G.; Umesh, K., Computational Studies Towards Identification of Lead Herbal Compounds of Medicinal Importance for Development of Nutraceutical Against COVID-19. 2020.

10. Raja Sankar, S., Bio-Chemical Extraction of Active Compounds of Agaricus (Mushrooms) and it's Antioxidant Activity. 2015, 14, 36-39.

11. Elkhateeb, W.; Daba, G.; Thomas, P.; Wen, T.-C., Medicinal mushrooms as a new source of natural therapeutic bioactive compounds. 2019, 18 (2), 88-101.

12. Sánchez, C., Bioactives from Mushroom and Their Application. 2017; pp 23-57.

13. Deshmukh, S. K.; Prakash, V.; Ranjan, N., Marine Fungi: A Source of Potential Anticancer Compounds. 2018, 8 (2536).

14. Gomes, A. R.; Duarte, A. C.; Rocha-Santos, T. A. P., Analytical Techniques for Discovery of Bioactive Compounds from Marine Fungi. In Fungal Metabolites, Mérillon, J.M.; Ramawat, K. G., Eds. Springer International Publishing: Cham, 2017; pp 415-434.

15. Hasan, S.; Ansari, M. I.; Ahmad, A.; Mishra, M., Major bioactive metabolites from marine fungi: A Review. Bioinformation 2015, 11, 176-181.

16. Daina, A.; Michielin, O.; Zoete, V., SwissADME: a free web tool to evaluate pharmacokinetics, drug-likeness and medicinal chemistry friendliness of small molecules. Sci Rep 2017, 7, 42717-42717.

17. Morris, G. M.; Huey, R.; Lindstrom, W.; Sanner, M. F.; Belew, R. K.; Goodsell, D. S.; Olson, A. J., AutoDock4 and AutoDockTools4: Automated docking with selective receptor flexibility. Journal of computational chemistry 2009, 30 (16), 2785-91.

18. Fuhrmann, J.; Rurainski, A.; Lenhof, H. P.; Neumann, D., A new Lamarckian genetic algorithm for flexible ligand-receptor docking. Journal of computational chemistry 2010, 31 (9), 1911-8.

19. Wang, Q.; Zhang, Y.; Wu, L.; Niu, S.; Song, C.; Zhang, Z.; Lu, G.; Qiao, C.; Hu, Y.; Yuen, K. Y.; Wang, Q.; Zhou, H.; Yan, J.; Qi, J., Structural and Functional Basis of SARS-CoV-2 Entry by Using Human ACE2. Cell 2020, 181 (4), 894-904.e9. 
20. Abraham, M. J.; Murtola, T.; Schulz, R.; Páll, S.; Smith, J. C.; Hess, B.; Lindahl, E., GROMACS: High performance molecular simulations through multi-level parallelism from laptops to supercomputers. SoftwareX 2015, 1-2, 19-25.

21. Huang, J.; Rauscher, S.; Nawrocki, G.; Ran, T.; Feig, M.; de Groot, B. L.; Grubmüller, H.; MacKerell, A. D., Jr., CHARMM36m: an improved force field for folded and intrinsically disordered proteins. Nat Methods 2017, 14 (1), 71-73.

22. Mark, P.; Nilsson, L., Structure and Dynamics of the TIP3P, SPC, and SPC/E Water Models at 298 K. The Journal of Physical Chemistry A 2001, 105 (43), 9954-9960.

23. Kräutler, V.; van Gunsteren, W. F.; Hünenberger, P. H., A fast SHAKE algorithm to solve distance constraint equations for small molecules in molecular dynamics simulations. Journal of computational chemistry 2001, 22 (5), 501-508.

24. Kadoura, A.; Salama, A.; Sun, S., Switching Between the NVT and NpT Ensembles Using the Reweighting and Reconstruction Scheme. Procedia Computer Science 2015, 51, 1259-1268.

25. Petersen, H. G., Accuracy and efficiency of the particle mesh Ewald method. The Journal of Chemical Physics 1995, 103 (9), 3668-3679.

26. Laskowski, R.; Macarthur, M. W.; Moss, D. S.; Thornton, J., PROCHECK: A program to check the stereochemical quality of protein structures. Journal of Applied Crystallography 1993, 26, 283-291.

27. Heinig, M.; Frishman, D., STRIDE: a web server for secondary structure assignment from known atomic coordinates of proteins. Nucleic Acids Res 2004, 32 (Web Server issue), W500-W502. 